\title{
Clinicopathological significance of claudin 4 expression in gastric carcinoma: a systematic review and meta-analysis
}

This article was published in the following Dove Press journal:

OncoTargets and Therapy

27 May 2016

Number of times this article has been viewed

\author{
Xiaowan Chen ${ }^{1, *}$ \\ Junhua Zhao',* \\ Ailin $\mathrm{Li}^{1,2}$ \\ Peng Gao' \\ Jingxu Sun' \\ Yongxi Song' \\ Jingjing Liu' \\ Ping Chen' \\ Zhenning Wang' \\ 'Department of Surgical Oncology \\ and General Surgery, ${ }^{2}$ Department of \\ Radiation Oncology, First Hospital of \\ China Medical University, Shenyang, \\ People's Republic of China \\ *These authors contributed equally \\ to this work
}

Correspondence: Zhenning Wang Department of Surgical Oncology and General Surgery, First Hospital of China Medical University, I 55 North Nanjing Street, Heping District, Shenyang I I000I, People's Republic of China

Tel +862483283556

Fax +862483283556

Email josieon826@sina.cn
Background: The prognostic significance of claudin 4 (CLDN4) in patients with gastric cancer (GC) is controversial. This meta-analysis aims to assess the correlation between CLDN4 expression and clinicopathological characteristics and assess the prognostic significance of CLDN4 in GC.

Methods: We searched the PubMed and Embase databases. We performed the meta-analysis with odds ratio (OR), hazard ratio (HR), and 95\% confidence interval (CI) as effect values.

Results: Fourteen studies containing 2,106 patients with GC were analyzed. The overall analysis showed that CLDN4 expression was associated with increasing $\mathrm{pT}$ category, tumor size, and lymph node metastasis in patients with GC (pT3-T4 vs pT1-T2: OR $=1.56,95 \%$ $\mathrm{CI}=1.13-2.16 ; P<0.01$; large tumor size vs small tumor size: $\mathrm{OR}=1.64,95 \% \mathrm{CI}=1.15-2.34$; $P<0.01$; positive lymph node metastasis vs negative lymph node metastasis: $\mathrm{OR}=1.49,95 \%$ $\mathrm{CI}=1.12-1.97 ; P<0.01)$. CLDN4 expression was associated with histological differentiation (differentiated type vs undifferentiated type: $\mathrm{OR}=2.90,95 \% \mathrm{CI}=1.32-6.37 ; P=0.01$; Lauren intestinal type vs diffuse type: $\mathrm{OR}=3.51,95 \% \mathrm{CI}=1.48-8.28 ; P<0.01)$. CLDN4 expression was also strongly associated with sex and age. This meta-analysis found no significant association between CLDN4 expression and prognosis for overall survival in patients with $\mathrm{GC}$ ( $\mathrm{HR}=0.74$, $95 \%$ CI $=0.43-1.27 ; P=0.28)$.

Conclusion: Present study indicates that aberrant CLDN4 expression plays an important role in the clinicopathological characteristics of GC.

Keywords: CLDN4, gastric cancer, biomarker, meta-analysis, prognosis, metastasis, stage

\section{Introduction}

Despite a recent reduction in the incidence of gastric cancer (GC), it remains the second most frequent cause of cancer-related deaths, leading to 723,100 deaths worldwide in 2012. ${ }^{1}$ Although the clinical prognosis for GC has been improved by the development of surgical techniques and adjuvant chemoradiotherapy, most cases are at an advanced stage at the time of diagnosis and treatment options are limited. ${ }^{2}$ Moreover, using molecular subtypes to predict and provide information for treatment of patients with GC leaves much to be desired. The identification and characterization of novel pathways and distinct molecular markers that are exploitable for diagnosis and therapy remains a challenging, but crucial endeavor to make progress in improving GC prognosis.

Claudins are located at the most apical component of intercellular cell-cell junctions, where they establish cell polarity. ${ }^{3}$ Claudin 4 (CLDN4) is a member of claudin family, which consists of 209 amino acids and encodes four putative transmembrane segments. ${ }^{4,5}$ Gress et al $^{6}$ firstly reported that CLDN4 overexpression occurs in pancreatic 
cancer. High levels of CLDN4 have been described in multiple other cancer entities, such as esophageal, ${ }^{7}$ ovarian, endometrial, ${ }^{8}$ bladder, renal, and prostate cancer. ${ }^{9}$

Numerous studies have demonstrated aberrant expression of claudins in several human cancers. Among the various claudin proteins related to GC, the function of CLDN4 is not consistent. For example, Ohtani et $\mathrm{al}^{10}$ found that the expression of CLDN4 significantly correlated with favorable survival in patients with $\mathrm{GC}$, while Resnick et $\mathrm{al}^{11}$ reported that moderate to strong CLDN4 staining in GC was significantly associated with poor survival. Thus, further investigations are required to clarify these controversial results and the real functions of CLDN4 in GC. We performed this meta-analysis to investigate the association between CLDN4 expression and its clinicopathological and prognostic significance in GC.

\section{Methods}

\section{Search strategy}

All relevant articles were retrieved by searching the PubMed and Embase databases using different combinations of the terms "claudin 4", "claudin-4", "claudin4", "CLDN4", "CLDN 4", "CLDN-4", and "gastric cancer". The reference lists of the retrieved studies and reviews were perused manually to check for potentially relevant studies. Two authors (XC and JZ) carried out the search independently.

\section{Study eligibility criteria}

Studies were considered eligible if they met all of the following criteria: 1) the patients enrolled were all diagnosed with GC and investigated for CLDN4 expression status; 2) the prognostic and clinicopathological significance of CLDN4 expression on at least one of the outcome measures of interest was assessed; 3 ) the outcome measures of interest were reported in the study or calculated from the published data; and 4) when several studies were based on the same patient population, only the most informative study was included.

\section{Data extraction and quality assessment}

The following information was recorded for each study: the first author's name, the study country or area, the sample size, clinicopathological parameters, detection method, and prognostic factors.

Data collection and assessment followed the recommendations of the Preferred Reporting Items for Systematic Reviews and Meta-analyses guideline. ${ }^{12}$ The data were extracted by two investigators (XC and JZ) independently. We used the Newcastle-Ottawa scale specific to cohort study to assess all included studies. ${ }^{13}$ Discrepancies were discussed by all investigators to reach a consensus.

\section{Statistical methods}

We used odds ratio (OR) and hazard ratio (HR) and their $95 \%$ confidence interval $(\mathrm{CI})$ as outcomes. ORs were used to assess the relationship between the expression of CLDN4 and clinicopathological features. HR was used to summarize the association between the expression of CLDN4 and GC survival. If the HR and its $95 \%$ CI were not provided in the study directly, these values were calculated from the available data using the method designed by Tierney et al. ${ }^{14}$

Heterogeneity among the studies was evaluated using Cochran's $Q$ test and the $I^{2}$ statistic. Values of $P<0.10$ for the $Q$ statistic and/or $I^{2}>50 \%$ were considered to show statistically significant heterogeneity. A random-effects model was used if there was significant heterogeneity; otherwise, a fixed-effects model was used. Publication bias was evaluated using a funnel plot.

Statistical analysis was performed using Review Manager 5.2 software (Copenhagen: The Nordic Cochrane Centre; The Cochrane Collaboration, 2012). A two-sided $P$-value of $<0.05$ was considered statistically significant for all tests.

\section{Results}

\section{Characteristics of the studies included}

We identified 64 studies in this systematic literature search. A total of 43 potential studies were retrieved after duplicates were removed. Twenty-nine studies were then excluded because they lacked outcomes of interest. The remaining 14 studies ${ }^{10,11,15-26}$ met the selection criteria for the final analysis (Figure 1). These studies were from the People's Republic of China, Korea, Taiwan, Japan, Finland, and USA, contained 2,106 patients with GC,

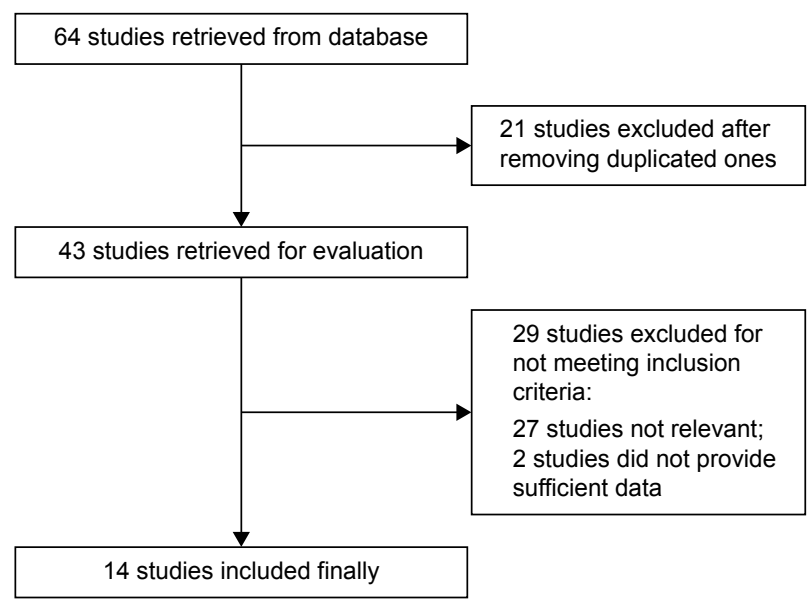

Figure I Flow diagram showing the selection process for the included studies. 
Table I Baseline characteristics of the included studies

\begin{tabular}{|c|c|c|c|c|c|c|c|c|}
\hline Study (year) & Countrylarea & Patients & $\begin{array}{l}\text { AB } \\
\text { source }\end{array}$ & AB type & Dilution & Follow-up & $\begin{array}{l}\text { Cut-off point } \\
\text { (high/positive), \% }\end{array}$ & Score \\
\hline Wang $(2015)^{15}$ & People's Republic of China & 92 & Rabbit & Polyclonal & NR & NR & $\geq 10$ & 3 \\
\hline Tokuhara $(2015)^{16}$ & Japan & 94 & Rabbit & Polyclonal & $\mathrm{I}: 200$ & NR & $\geq 25$ & 7 \\
\hline Zhu et al $(2013)^{17}$ & People's Republic of China & 329 & Mouse & Monoclonal & $1: 100$ & $56(I-136)$ & $>75$ & 7 \\
\hline Kwon et al $(201 \mathrm{I})^{18}$ & Korea & 485 & Mouse & Monoclonal & $\mathrm{I}: 40$ & 150 & NR & 5 \\
\hline Jung et al $(2011)^{19}$ & Korea & 72 & NR & NR & $\mathrm{I}: 200$ & $36.8(2.7-48.8)$ & $\geq 25$ & 6 \\
\hline Hwang et al $(2010)^{20}$ & Taiwan & 189 & Mouse & Monoclonal & $1: 100$ & 60 & $\geq 10$ & 7 \\
\hline Ohtani et al (2009) 10 & Japan & 124 & Mouse & Monoclonal & $\mathrm{I}: 100$ & $22.2(1-64.37)$ & $>50$ & 5 \\
\hline Lee et al $(2008)^{2 !}$ & Taiwan & 88 & Mouse & Monoclonal & $\mathrm{I}: 100$ & NR & $\geq 10$ & 4 \\
\hline Matsuda et al $(2007)^{22}$ & Japan & 94 & Mouse & Monoclonal & $\mathrm{I}: 100$ & NR & $>30$ & 3 \\
\hline Soini et al $(2006)^{23}$ & Finland & 118 & Mouse & Monoclonal & $\mathrm{I}: 50$ & NR & NR & 5 \\
\hline Kuo et al $(2006)^{24}$ & Taiwan & 93 & Goat & Polyclonal & NR & NR & $\geq 10$ & 4 \\
\hline Cunningham et al $(2006)^{25}$ & USA & 133 & Mouse & Monoclonal & $\mathrm{I}: 500$ & NR & $\geq 10$ & 4 \\
\hline Resnick et al (2005) ${ }^{\prime \prime}$ & USA & 146 & Mouse & Monoclonal & $\mathrm{I}: 500$ & $34(12-180)$ & NR & 4 \\
\hline Lee et al $(2005)^{26}$ & Korea & 49 & Mouse & Monoclonal & $\mathrm{I}: 500$ & NR & NR & 5 \\
\hline
\end{tabular}

Abbreviations: $A B$, antibody; NR, not reported.

and were published between 2005 and 2015. Their basic characteristics and study quality are summarized in Table 1.

\section{Correlation of CLDN4 expression with clinicopathological parameters \\ CLDN4 expression and tumor-node-metastasis stage}

The ORs for $\mathrm{pT}$ categories were available in six studies, including 513 cases with pT3-4 categories and 320 cases with
pT1-2 categories. Our results show that CLDN4 expression was significantly higher in patients with GC of high $\mathrm{pT}$ category than in those of low pT category ( $\mathrm{OR}=1.56,95 \%$ $\mathrm{CI}=1.13-2.16 ; P<0.01$ ) (Table 2; Figure 2A). Moreover, the OR for lymph node metastasis was available in nine studies, including 670 patients with GC with positive lymph node metastasis and 400 patients with GC with negative lymph node metastasis. The pooled OR indicates that CLDN4 expression was significantly higher in patients with GC with

Table 2 Detailed results for clinicopathological and prognostic significance of CLDN4 expression

\begin{tabular}{|c|c|c|c|c|}
\hline Parameters & Effect values (OR/HR) & $95 \% \mathrm{Cl}$ & $P$-value & Heterogeneity $\left(I^{2}\right), \%$ \\
\hline \multicolumn{5}{|c|}{ Clinicopathological characteristics } \\
\hline $\begin{array}{l}\text { PT category } \\
\text { T3-T4 versus TI-T2 }\end{array}$ & 1.56 & $1.13-2.16$ & $<0.01$ & 6.00 \\
\hline $\begin{array}{l}\text { Lymph node metastasis } \\
\text { Positive versus negative }\end{array}$ & 1.49 & $1.12-1.97$ & $<0.01$ & 37.00 \\
\hline $\begin{array}{l}\text { Stage } \\
\text { III-IV versus I-II }\end{array}$ & 0.99 & $0.54-1.82$ & 0.97 & 69.00 \\
\hline $\begin{array}{l}\text { Differentiation } \\
\text { Differentiated versus undiffe }\end{array}$ & 2.90 & $1.32-6.37$ & $<0.01$ & 85.00 \\
\hline $\begin{array}{l}\text { Lauren type } \\
\text { Intestinal versus diffuse }\end{array}$ & 3.51 & $1.48-8.28$ & $<0.01$ & 76.00 \\
\hline $\begin{array}{l}\text { Lymphatic invasion } \\
\text { Positive versus negative }\end{array}$ & 1.06 & $0.39-2.89$ & 0.91 & 82.00 \\
\hline \multicolumn{5}{|l|}{ Positive versus negative } \\
\hline $\begin{array}{l}\text { Age } \\
\text { Older versus younger }\end{array}$ & 1.50 & $1.13-1.99$ & $<0.01$ & 36.00 \\
\hline \multicolumn{5}{|l|}{ Males versus females } \\
\hline $\begin{array}{l}\text { Tumor size } \\
\text { Large versus small }\end{array}$ & 1.64 & $1.15-2.34$ & $<0.01$ & 44.00 \\
\hline \multicolumn{5}{|l|}{ Prognosis } \\
\hline HR for OS total & 0.74 & $0.43-1.27$ & 0.28 & 81.00 \\
\hline HR for OS in Asian subgroup & 0.58 & $0.33-1.05$ & 0.07 & 80.00 \\
\hline HR for OS in white subgroup & 2.07 & $|.22-3.5|$ & $<0.01$ & Not applicable \\
\hline
\end{tabular}

Abbreviations: CLDN4, claudin 4; HR, hazard ratio; OR, odds ratio; OS, overall survival; $\mathrm{Cl}$, confidence interval. 


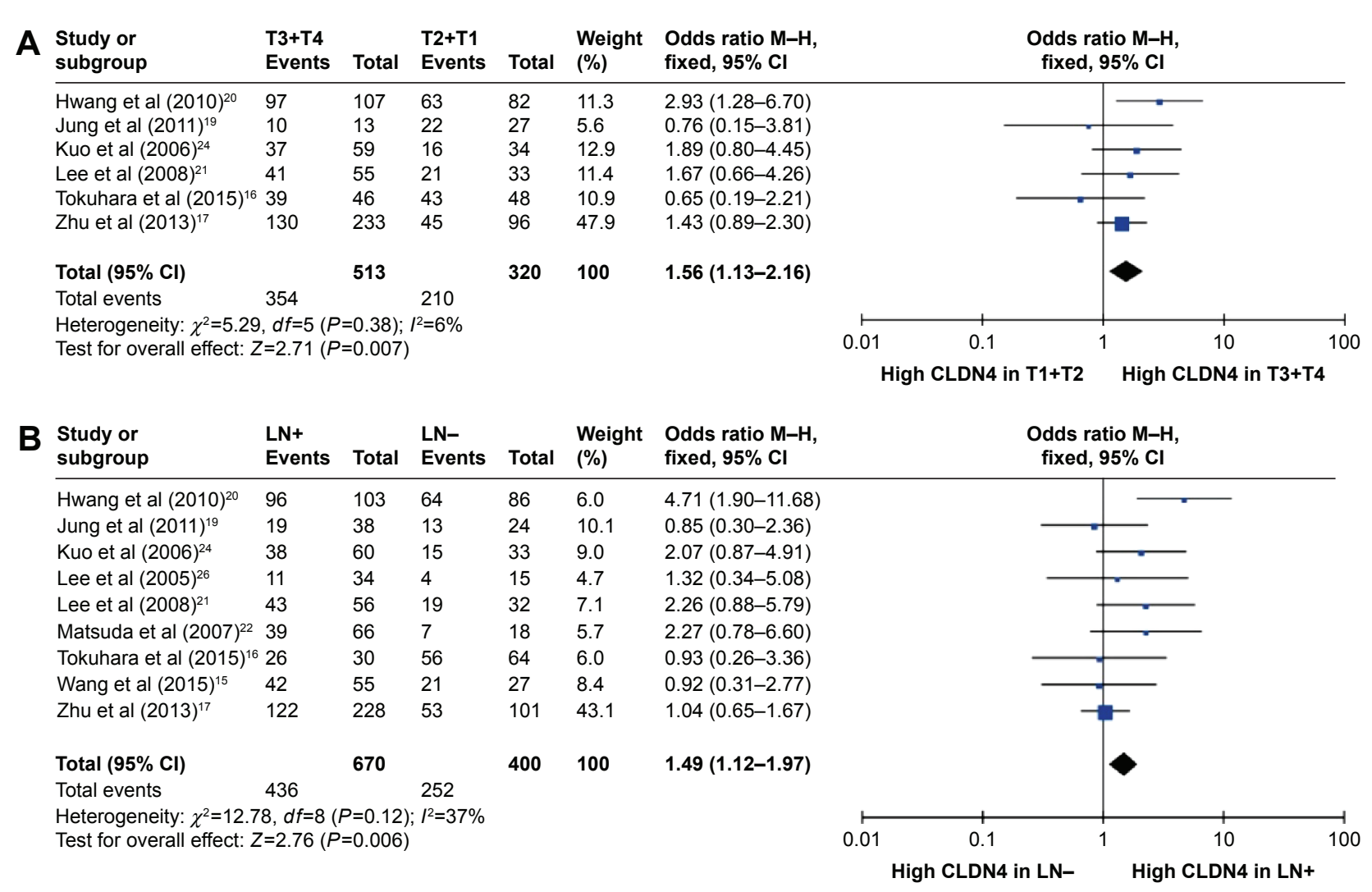

Figure 2 Pooled analysis for the association between CLDN4 expression and TNM stage.

Notes: (A) Pooled analysis for the association between CLDN4 expression and pT category. (B) Pooled analysis for the association between CLDN4 expression and lymph node metastasis.

Abbreviations: $\mathrm{Cl}$, confidence interval; CLDN4, claudin 4; $d f$, degrees of freedom; LN+, positive lymph node metastasis; LN-, negative lymph node metastasis; TNM, tumornode-metastasis; $\mathrm{M}-\mathrm{H}$, Mantel-Haenszel.

lymph node metastasis $(\mathrm{OR}=1.49,95 \% \mathrm{CI}=1.12-1.97$; $P<0.01$ ) (Table 2; Figure 2B).

Including six studies with 438 early stage (I-II) patients with GC and 436 advanced stage (III-IV) patients with $\mathrm{GC}$, there was no significant association between CLDN4 expression and GC stage (OR $=0.99,95 \% \mathrm{CI}=0.54-1.82$; $P=0.97$ ) (Table 2).

\section{CLDN4 expression and the histologic type}

The pooled OR, including 499 differentiated GC and 692 undifferentiated GC from ten studies, is shown in Table 2, indicating that CLDN4 expression was strongly associated with histological type in GC and high CLDN4 expression was more inclined to appear in differentiated $\mathrm{GC}(\mathrm{OR}=2.90$, 95\% CI $=1.32-6.37 ; P=0.01$ ).

Furthermore, pooled OR from six studies, including 259 intestinal type patients with GC and 274 diffuse type GC, suggests that aberrant CLDN4 expression was significantly higher in Lauren intestinal type GC than that in Lauren diffuse type $\mathrm{GC}(\mathrm{OR}=3.51,95 \% \mathrm{CI}=1.48-8.28 ; P<0.01)$ (Table 2).
CLDN4 expression and venous and lymphatic invasion

The pooled OR from five studies is shown in Table 2, indicating that aberrant CLDN4 expression was not significantly higher in positive lymphatic invasion than that in negative lymphatic invasion $(\mathrm{OR}=1.06,95 \% \mathrm{CI}=0.39-2.89 ; P=0.91)$. The pooled OR from four studies, including 170 patients with GC with positive venous invasion and 281 patients with GC with negative venous invasion, is shown in Table 2, indicating that CLDN4 expression was not associated with venous invasion in patients with GC (OR $=1.11,95 \%$ $\mathrm{CI}=0.52-2.37 ; P=0.79$ ).

\section{CLDN4 expression and age, sex, and tumor size}

We looked at the relationship between age and CLDN4 expression. The pooled OR from seven studies, including 529 older patients with GC and 450 younger patients with GC, is shown in Table 2. Our results show that CLDN4 expression was significantly higher in older patients with $\mathrm{GC}$ than in younger ones $(\mathrm{OR}=1.50,95 \% \mathrm{CI}=1.13-1.99$; $P<0.01)$. 
Next, we assessed the association between CLDN4 and sex. The pooled OR from eight studies, including 672 males and 356 females, is shown in Table 2 and indicates that CLDN4 expression was higher in males than in females ( $\mathrm{OR}=1.50,95 \% \mathrm{CI}=1.13-2.00 ; P<0.01)$.

Finally, CLDN4 expression also appears to be associated with tumor size. The pooled OR from three studies is shown in Table 2 and shows that aberrant CLDN4 expression was significantly higher in larger tumors than in smaller tumors $(\mathrm{OR}=1.64,95 \% \mathrm{CI}=1.15-2.34 ; P<0.01)$.

\section{Correlation of CLDN4 expression with prognostic effect}

In a pooled analysis including all studies ${ }^{10,11,17-20}$ with data on prognostic effect for CLDN4 expression in GC, we found no significant association between CLDN4 expression and prognosis for overall survival in patients with $\mathrm{GC}(\mathrm{HR}=0.74$, 95\% CI $=0.43-1.27 ; P=0.28)$, with huge heterogeneity $\left(I^{2}=81 \%, P<0.01\right)$. In subgroup analysis by ethnic group, we found that there was a different trend between Asian group and Caucasian group. Highly expressed CLDN4 tended to have better prognosis in Asian group without significance ( $\mathrm{HR}=0.58,95 \% \mathrm{CI}=0.33-1.05 ; P=0.07)$, while it showed association with poor prognosis in Caucasian group with significance $(\mathrm{HR}=2.07,95 \% \mathrm{CI}=1.22-3.51 ; P=0.007)$ (Table 2; Figure 3).

\section{Discussion}

CLDN4 is an integral membrane protein that belongs to the claudin family. The claudin family consists of approximately
23 proteins that are essential for the formation of tight junctions (TJs) in epithelial and endothelial cells. The exact combination of claudin proteins within a given tissue is thought to determine the selectivity and strength of the TJs. Recent gene expression profiling analyses have shown that claudin gene expression is frequently altered in various cancers. Specifically, CLDN1, 3, 4, 5, 7, 10, and 16 have been found altered in various cancers. The overexpression of these proteins in cancer (which typically lose their TJs) is unexpected, but may be related to roles that are unrelated to TJ formation. For instance, CLDN4 is overexpressed in several tumors and has been suggested to have roles in cell proliferation, motility, invasion, and survival. However, Li et $\mathrm{al}^{26}$ suggested a novel function for CLDN4 in cancer through its ability to reorganize the cellular environment to favor angiogenesis. By using both in vitro and in vivo assays, $\mathrm{Li}$ et al found that CLDN4 expression led to significant changes in gene expression. Also, these changes were accompanied by functional changes in angiogenesis. CLDN4 is the core protein to form the $\mathrm{TJ}$, which plays an important role in cell adhesion. Its aberrant expression was detected in various cancers, while its expression and functions in GC still remain unclear and the clinical significance of CLDN4 expression in GC has not been thoroughly investigated. Therefore, we performed this meta-analysis to systematically evaluate the association among CLDN4 expression and clinicopathological features and prognostic factors in GC.

The overall analysis showed that CLDN4 expression was significantly higher in large tumor group, pT3-4 categories, and positive lymph node metastasis group, while CLDN4

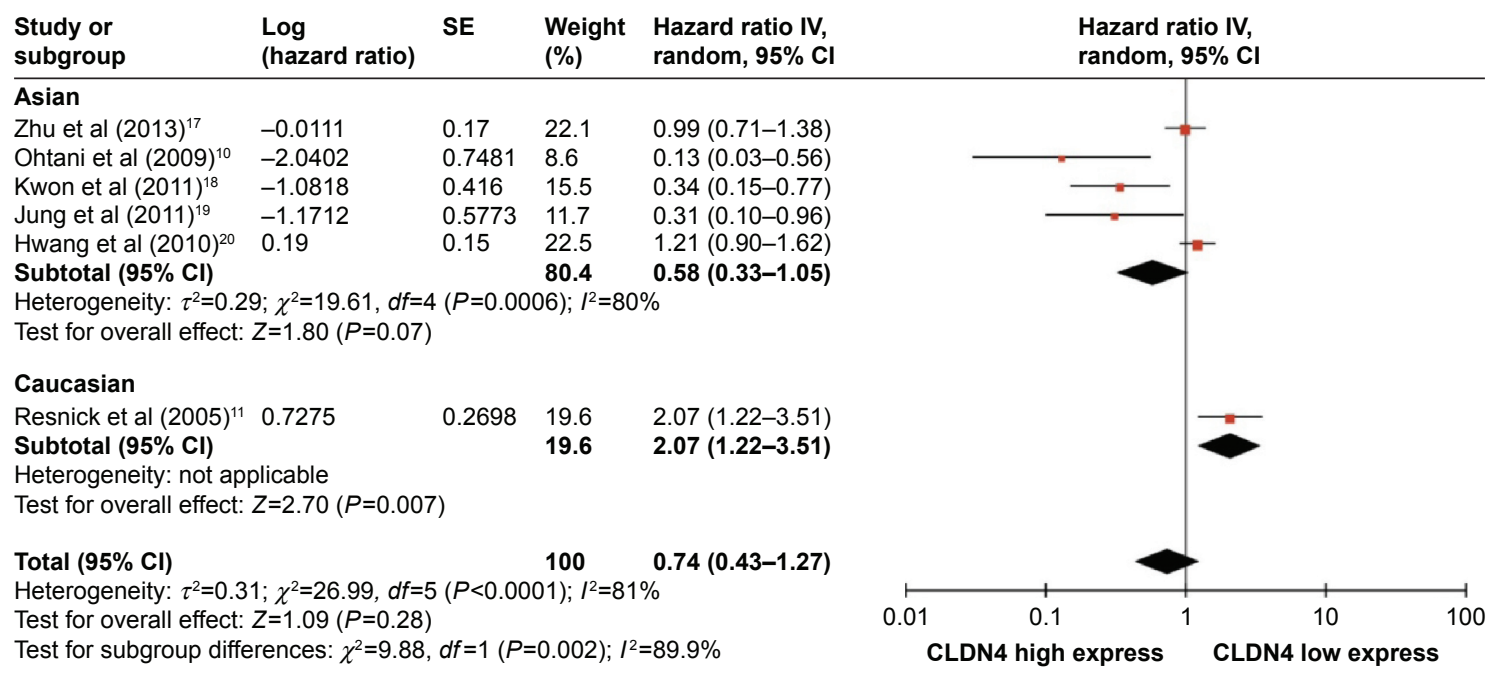

Figure 3 Correlation of CLDN4 expression with prognostic effect.

Notes: There was a different trend between Asian group and Caucasian group. High CLDN4 expression tended to have better prognosis in Asian group without significance, while it showed association with poor prognosis in Caucasian group with significance.

Abbreviations: $\mathrm{Cl}$, confidence interval; CLDN4, claudin 4; df, degrees of freedom; SE, standard error; IV, inverse variance. 
expression was also significantly higher in patients with GC of differentiated histological type and intestinal type of Lauren. Moreover, CLDN4 expression was strongly associated with sex and age in patients with GC. However, CLDN4 expression had no significant association with venous or lymphatic invasion. In the aspect of relationship between CLDN4 expression and prognosis of GC, we found that there was no significant association between CLDN4 expression and overall survival of patients with GC.

This meta-analysis revealed that CLDN4 played a dual role in the relationship between its increased expression and the clinicopathological features of GC. Elevated CLDN4 expression was found in well-differentiated or moderately differentiated GC and intestinal type GC. This phenomenon was in according with the intercellular cell-cell junctions function of CLDN4. However, we also found that high expression of CLDN4 significantly contributed to the invasive and metastatic phenotype of GC, such as advanced pT category, lymph node metastasis, and larger tumor size. The results of the meta-analysis suggest that the role CLDN4 plays in GC progression is apparently in contradiction to its physiological role as a cell-cell adhesion molecule. These results have been corroborated in ovarian cancer. ${ }^{28}$

For every plus, there is a minus. In the aspect of association between CLDN4 expression and GC prognosis, the results were controversial. Of the previous studies, Tsutsumi et $\mathrm{al}^{29}$ reported that increased expression of CLDN4 predicts better prognosis in pancreatic ductal adenocarcinoma. Hsueh et $\mathrm{al}^{30}$ indicated that decreased CLDN4 expression independently predicted shorter distant metastasis-free survival of nasopharyngeal cancer. These results were all presented in Asian group and are in line with our meta-analysis subgroup results. While in the Caucasian group, the results showed that high CLDN4 expression predicted poor prognosis in patients with GC. The controversial finding might not only be due to the difference between ethnic groups, but also caused by the dual role of CLDN4 expression in the invasion process of GC. During the invasion process, loss of intercellular adhesion is one of the early critical steps toward metastasis, and reduced expression of CLDN4 correlates with the loss of differentiation, which predicts worse prognosis of GC. Meanwhile, during the metastatic process, increased cell-to-cell adhesions formed by TJs lead to the planting of cancer cells into the metastatic organ, and high CLDN4 expression is related to facilitating the metastatic potential, which indicates poor prognosis of patients with GC. Therefore, we suppose the dual role of CLDN4 expression has led to the controversial results in the prognosis and survival analysis among studies.
Thus, it is of more significance to focus on the role of CLDN4 in individual processes during cancer development.

Several studies have tried to elucidate the molecular mechanisms by which CLDN4 impacts tumor progression and survival. The potential explanation may be that aberrant CLDN4 expression influences the expression of matrix metalloproteinases (MMPs), which play crucial roles in tumor invasion and tumor spread. Hwang et $\mathrm{al}^{20}$ suggested that CLDN4 affects the expression and activity of MMP-2 and MMP-9 either directly or by modulating signal transduction, and that these two proteins stimulate tumor cell invasion in GC. Prior to that study, Lee et $\mathrm{al}^{21}$ found that CLDN4 expression was significantly associated with MMP-9 expression, but not with MMP-2 expression. Moreover, Agarwal et $\mathrm{a}^{28}$ confirmed that CLDN4 overexpression enhanced invasion and increased migration, and was associated with increased MMP-2 activity, through their experiments in ovarian cancer cell lines. In addition, Miyamori et al ${ }^{31}$ showed that claudin promotes activation of pro-MMP-2 mediated by membrane-type MMP, which indicates the modulatory effects of claudins on MMP-2 activation. They suggested that claudin recruits all membrane-type MMPs and pro-MMP-2 on the cell surface to achieve elevated focal concentrations and, consequently, enhances activation of pro-MMP-2.

Regardless of their exact functions in cancer cells, claudin protein expression may have significant clinical relevance. Of particular interest is the possible use of Clostridium perfringens enterotoxin (CPE) as a novel chemotherapeutic compound. CPE is a natural ligand for CLDN4 proteins, and binding of the toxin to CLDN4 leads to a rapid cytolysis of the cells. ${ }^{32-34}$ Thus, high expression of CLDN4 in cancer might represent a unique opportunity for innovative therapy using CPE. ${ }^{35}$ Indeed, CLDN4 has been shown to be sensitive to CPE-mediated cytolysis in pancreatic, ${ }^{36}$ breast, ${ }^{37}$ and ovarian cancer. ${ }^{38}$ Importantly, these studies showed that no significant toxicity was encountered in mice upon intratumoral CPE treatment. These findings are important because the unusual expression patterns of CLDN4 suggest utility for detection, diagnosis, and treatment of GC.

Previously, there was a meta-analysis regarding on the correlation of CLDN4 expression with clinic pathological characteristic and prognosis of GC. ${ }^{39}$ In that meta-analysis they showed over expression of CLDN4 was only associated with poor survival, advanced stage and lymphoid node metastasis. In our meta-analysis, we found a significant association between expression of CLDN4 and lymph node metastasis, pT category, tumor size, histological differentiation, sex, and age, but did not find a significant relationship 
of CLDN4 expression with either advanced stage or survival in patients with GC. The differences of these two studies might result in different studies included and different data extracted. Comparing with the previous meta-analysis, we have analyzed six studies published before ${ }^{18,21-23,25,26}$ and added two studies published recently. ${ }^{15,16}$ All studies we included were published in English. In addition, we had to write to some authors for individual data to make pooled analysis more confirmed and convincible. For instance, Zhu et $\mathrm{al}^{16}$ presented the results of GC prognosis in subgroup, but the results were not calculated for all patients. Therefore, we consulted the author, and received the data and also obtained permission of using the unpublished results. Moreover, regarding the relationship between CLDN4 expression and GC clinicopathological characteristics, we found more significant association than the previous meta-analysis, which has made our meta-analysis more constructive and advisable.

There are several limitations in this meta-analysis. First, this meta-analysis was based on published data from retrospective studies and we could not obtain individual data. Second, heterogeneity could not be ignored and we used the random-effects model if there was considerable heterogeneity. Moreover, limited number of available studies impacted the statistical power of subgroup analysis.

\section{Conclusion}

The present study indicates that aberrant CLDN4 expression plays an important role in the clinicopathological characteristics of GC. Further large-scale studies, especially multicenter and well-matched cohort research, will provide more insight into the role of CLDN4 in the prognosis and clinical implementation of patients with GC.

\section{Acknowledgments}

The authors thank the Department of Surgical Oncology of First Hospital of China Medical University and the College of China Medical University for technical assistance in this analysis. This work was supported by National Science Foundation of China (No 81402520), Natural Science Foundation of Liaoning Province (No 2014029201), and Program of Education Department of Liaoning Province (L2014307).

\section{Author contributions}

All authors contributed toward data analysis, drafting and revising the paper and agree to be accountable for all aspects of the work.

\section{Disclosure}

The authors report no conflicts of interest in this work.

\section{References}

1. Torre LA, Bray F, Siegel RL, Ferlay J, Lortet-Tieulent J, Jemal A. Global cancer statistics, 2012. CA Cancer J Clin. 2015;65(2):87-108.

2. DeSantis CE, Lin CC, Mariotto AB, et al. Cancer treatment and survivorship statistics, 2014. CA Cancer J Clin. 2014;64(4):252-271.

3. Gumbiner B. Structure, biochemistry, and assembly of epithelial tight junctions. Am J Physiol. 1987;253(6 Pt 1):C749-C758.

4. Katahira J, Sugiyama H, Inoue N, Horiguchi Y, Matsuda M, Sugimoto N. Clostridium perfringens enterotoxin utilizes two structurally related membrane proteins as functional receptors in vivo. J Biol Chem. 1997; 272(42):26652-26658.

5. Morita K, Furuse M, Fujimoto K, Tsukita S. Claudin multigene family encoding four-transmembrane domain protein components of tight junction strands. Proc Natl Acad Sci U S A. 1999;96(2): 511-516.

6. Gress TM, Muller-Pillasch F, Geng M, et al. A pancreatic cancerspecific expression profile. Oncogene. 1996;13(8):1819-1830.

7. Montgomery E, Mamelak AJ, Gibson M, et al. Overexpression of claudin proteins in esophageal adenocarcinoma and its precursor lesions. Appl Immunohistochem Mol Morphol. 2006;14(1):24-30.

8. Gadducci A, Tana R, Cosio S, Fanucchi A, Genazzani AR. Molecular target therapies in endometrial cancer: from the basic research to the clinic. Gynecol Endocrinol. 2008;24(5):239-249.

9. Hayes MP, Ellenson LH. Molecular alterations in uterine serous carcinoma. Gynecol Oncol. 2010;116(2):286-289.

10. Ohtani S, Terashima M, Satoh J, et al. Expression of tight-junctionassociated proteins in human gastric cancer: downregulation of claudin-4 correlates with tumor aggressiveness and survival. Gastric Cancer. 2009;12(1):43-51.

11. Resnick MB, Gavilanez M, Newton E, et al. Claudin expression in gastric adenocarcinomas: a tissue microarray study with prognostic correlation. Hum Pathol. 2005;36(8):886-892.

12. Moher D, Liberati A, Tetzlaff J, Altman DG, PRISMA Group. Preferred reporting items for systematic reviews and meta-analyses: the PRISMA statement. PLoS Med. 2009;6(7):e1000097.

13. Stang A. Critical evaluation of the Newcastle-Ottawa scale for the assessment of the quality of nonrandomized studies in meta-analyses. Eur J Epidemiol. 2010;25(9):603-605.

14. Tierney JF, Stewart LA, Ghersi D, Burdett S, Sydes MR. Practical methods for incorporating summary time-to-event data into meta-analysis. Trials. 2007;8:16.

15. Wang H, Yang X. The expression patterns of tight junction protein claudin-1, -3, and -4 in human gastric neoplasms and adjacent non-neoplastic tissues. International Int J Clin Exp Pathol. 2015;8(1):881-887.

16. Tokuhara Y, Morinishi T, Matsunaga T, et al. Claudin-1, but not claudin-4, exhibits differential expression patterns between well- to moderately-differentiated and poorly-differentiated gastric adenocarcinoma. Oncol Lett. Jul 2015;10(1):93-98.

17. Zhu JL, Gao P, Wang ZN, et al. Clinicopathological significance of claudin-4 in gastric carcinoma. World J Surg Oncol. 2013;11:150.

18. Kwon MJ, Kim SH, Jeong HM, et al. Claudin-4 overexpression is associated with epigenetic derepression in gastric carcinoma. Lab Invest. 2011;91(11):1652-1667.

19. Jung H, Jun KH, Jung JH, Chin HM, Park WB. The expression of claudin-1, claudin-2, claudin-3, and claudin-4 in gastric cancer tissue. J Surg Res. 2011;167(2):e185-e191.

20. Hwang TL, Lee LY, Wang CC, Liang Y, Huang SF, Wu CM. Claudin-4 expression is associated with tumor invasion, MMP-2 and MMP-9 expression in gastric cancer. Exp Ther Med. 2010;1(5): 789-797.

21. Lee LY, Wu CM, Wang CC, et al. Expression of matrix metalloproteinases MMP-2 and MMP-9 in gastric cancer and their relation to claudin-4 expression. Histol Histopathol. 2008;23(5):515-521. 
22. Matsuda Y, Semba S, Ueda J, et al. Gastric and intestinal claudin expression at the invasive front of gastric carcinoma. Cancer Sci. 2007;98(7): 1014-1019.

23. Soini Y, Tommola S, Helin H, Martikainen P. Claudins 1, 3, 4 and 5 in gastric carcinoma, loss of claudin expression associates with the diffuse subtype. Virchow Arch. 2006;448(1):52-58.

24. Kuo WL, Lee LY, Wu CM, et al. Differential expression of claudin-4 between intestinal and diffuse-type gastric cancer. Oncol Rep. 2006; 16(4):729-734.

25. Cunningham SC, Kamangar F, Kim MP, et al. Claudin-4, mitogenactivated protein kinase kinase 4, and stratifin are markers of gastric adenocarcinoma precursor lesions. Cancer Epidemiol Biomarkers Prev. 2006;15(2):281-287.

26. Lee SK, Moon J, Park SW, Song SY, Chung JB, Kang JK. Loss of the tight junction protein claudin 4 correlates with histological growthpattern and differentiation in advanced gastric adenocarcinoma. Oncol Rep. 2005;13(2):193-199.

27. Li J, Chigurupati S, Agarwal R, et al. Possible angiogenic roles for claudin-4 in ovarian cancer. Cancer Biol Ther. 2009;8(19): $1806-1814$

28. Agarwal R, D'Souza T, Morin PJ. Claudin-3 and claudin-4 expression in ovarian epithelial cells enhances invasion and is associated with increased matrix metalloproteinase-2 activity. Cancer Res. 2005;65(16): 7378-7385.

29. Tsutsumi K, Sato N, Tanabe R, et al. Claudin-4 expression predicts survival in pancreatic ductal adenocarcinoma. Ann Surg Oncol. 2012; 19 Suppl 3:S491-S499.

30. Hsueh C, Chang YS, Tseng NM, et al. Expression pattern and prognostic significance of claudins 1,4 , and 7 in nasopharyngeal carcinoma. Hum Pathol. 2010;41(7):944-950.
31. Miyamori H, Takino T, Kobayashi Y, et al. Claudin promotes activation of pro-matrix metalloproteinase- 2 mediated by membrane-type matrix metalloproteinases. J Biol Chem. 2001;276(30):28204-28211.

32. Suzuki H, Kondoh M, Takahashi A, Yagi K. Proof of concept for claudintargeted drug development. Ann N Y Acad Sci. 2012;1258:65-70.

33. Neesse A, Griesmann H, Gress TM, Michl P. Claudin-4 as therapeutic target in cancer. Arch Biochem Biophys. 2012;524(1):64-70.

34. Maeda T, Murata M, Chiba H, et al. Claudin-4-targeted therapy using Clostridium perfringens enterotoxin for prostate cancer. Prostate. 2012; 72(4):351-360.

35. Michl P, Gress TM. Bacteria and bacterial toxins as therapeutic agents for solid tumors. Curr Cancer Drug Targets. 2004;4(8):689-702.

36. Michl P, Buchholz M, Rolke M, et al. Claudin-4: a new target for pancreatic cancer treatment using Clostridium perfringens enterotoxin. Gastroenterology. 2001;121(3):678-684.

37. Kominsky SL, Vali M, Korz D, et al. Clostridium perfringens enterotoxin elicits rapid and specific cytolysis of breast carcinoma cells mediated through tight junction proteins claudin 3 and 4. Am J Pathol. 2004; 164(5):1627-1633.

38. Santin AD, Cane S, Bellone S, et al. Treatment of chemotherapyresistant human ovarian cancer xenografts in C.B-17/SCID mice by intraperitoneal administration of Clostridium perfringens enterotoxin. Cancer Res. 2005;65(10):4334-4342.

39. Liu JX, Wei ZY, Chen JS, Lu HC, Hao L, Li WJ. Prognostic and clinical significance of claudin-4 in gastric cancer: a meta-analysis. World J Sur Oncol. 2015;13:207.
OncoTargets and Therapy

\section{Publish your work in this journal}

OncoTargets and Therapy is an international, peer-reviewed, open access journal focusing on the pathological basis of all cancers, potential targets for therapy and treatment protocols employed to improve the management of cancer patients. The journal also focuses on the impact of management programs and new therapeutic agents and protocols on

\section{Dovepress}

patient perspectives such as quality of life, adherence and satisfaction The manuscript management system is completely online and includes a very quick and fair peer-review system, which is all easy to use. Visit http://www.dovepress.com/testimonials.php to read real quotes from published authors. 